\title{
Interferon-related secretome from direct interaction between immune cells and tumor cells is required for upregulation of PD-L1 in tumor cells
}

Dear Editor,

PD-L1, also known as CD274, plays a vital role in tumor cell related immune escape. It can be expressed on the cell surface of many solid tumors (Brahmer et al., 2012) and inhibits $T$ cell proliferation and cytokine production by binding to the T cell surface receptor programmed death 1 (PD-1) or B7-1 (McClanahan et al., 2015). In 2013, targeting PD-1/ PD-L1 signaling for cancer immunotherapy was selected as the No.1 scientific breakthrough of the year by the editors of Science. Interferons (IFNs) are a group of pleiotropic cytokines, demonstrated anti-viral, anti-tumor, and immune regulatory functions (York et al., 2015). Type I interferon binds a heterodimeric receptor composed of IFNAR1 and IFNAR2. This activates a canonical JAK/STAT signaling pathway that ultimately induces a set of interferon-stimulated genes to exert its biological activity (Ejlerskov et al., 2015). Recently, PD-L1 was reported to be downstream of IFN signaling in human oral squamous carcinoma, melanoma, and human acute myeloid leukemia blast cells (Chen et al., 2012; Furuta et al., 2014; Kronig et al., 2014).

The tumor microenvironment plays an important role in tumor growth and metastasis. Different components of the tumor microenvironment such as T cells, B cells, NK cells, dendritic cells, mast cells, granulocytes, Treg cells, myeloid derived suppressor cells (MDSC), and tumor associated macrophages (TAM) are recruited by different pathways (Joyce and Fearon, 2015). Tumor cells have been shown to upregulate PD-L1 after interacting with infiltrating immune cells (Cho et al., 2011; Hou et al., 2014), but the mechanism by which this occurs is not well understood. In this study, we found that PD-L1 upregulation in tumors was dependent on direct interaction with immune cells and was driven by a secreted factor such as type I interferon after cell-cell contact.

Previous studies have demonstrated a positive correlation between tumor-infiltrating immune cells and elevated PD-L1 expression in tumor cells, but the mechanism by which this occurs is poorly understood. To investigate this, we co-cultured murine B16F10 melanoma cells with syngeneic splenocytes for $48 \mathrm{~h}$. In addition, to determine whether direct cell contact is required for immune cell-mediated PD-L1 expression, the two types of cells were separated by a transwell-membrane that blocked their direct cell-cell interactions. Furthermore, another condition was tested in which B16F10 cells and immune cells were co-cultured in the plate and B16F10 cells were cultured in the transwell insert (Fig. 1A). Then the non-adherent immune cells were removed and B16F10 cells were harvested and analyzed for PD-L1 expression by flow cytometry. PD-L1 was more highly expressed in B16F10 cells that were co-cultured with splenocytes than in those cultured alone (Fig. 1B). However, PD-L1 expression was not increased in B16F10 cells separated from the splenocytes by a transwell membrane. We also found that a B16F10-splenocyte co-culture was able to induce PD-L1 in tumor cells separated from the co-culture by a transwell membrane (Fig. 1B). These effects were also observed in PD-L1 mRNA level changes by qPCR (Fig. 1C). These results suggested that active factors were secreted into the supernatant after the direct cell-cell interaction that was able to induce PD-L1 expression in tumor cells.

To identify whether the regulation of PD-L1 was indeed driven by a secreted factor, B16F10 cells and splenocytes were co-cultured for $48 \mathrm{~h}$. The supernatant was collected and centrifuged, and then used to treat B16F10 cells independently. The corresponding supernatant derived from B16F10 cells and splenocytes alone was also used to treat B16F10 cells as control groups (Fig. 1D). After $24 \mathrm{~h}$, B16F10 cells treated with supernatant from the co-culture expressed more PD-L1 than cells treated with supernatant from the control mono-cultures (Fig. 1E and $1 \mathrm{~F}$ ). In addition, co-cultures of B16F10 cells with bone marrow (BM)-derived cells (Fig. $1 \mathrm{G}$ ) or lymph node (LN)-derived cells also upregulated PD-L1 expression (Fig. 1H). To determine whether a similar effect would be seen in other types of cancer cells, additional studies on MC38 and Hepa1-6 cells 
A

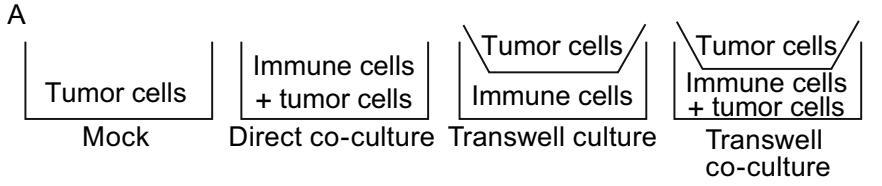

B

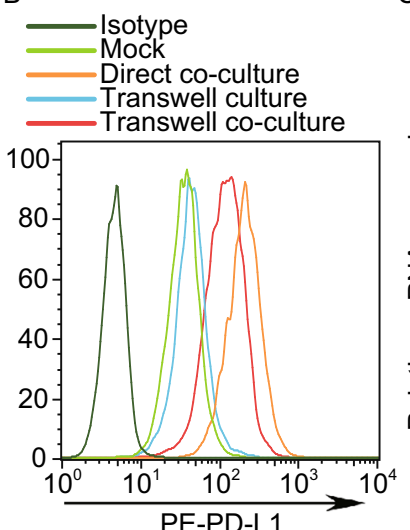

C Mock
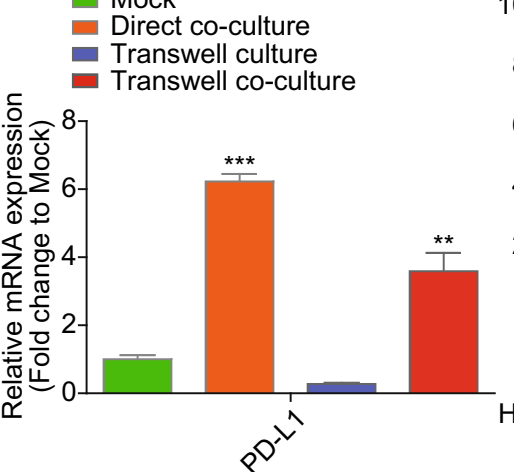

G

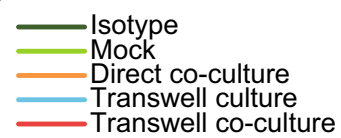

Isotype

Mock

Culture supernatant transfer

Co-culture supernatant transfer
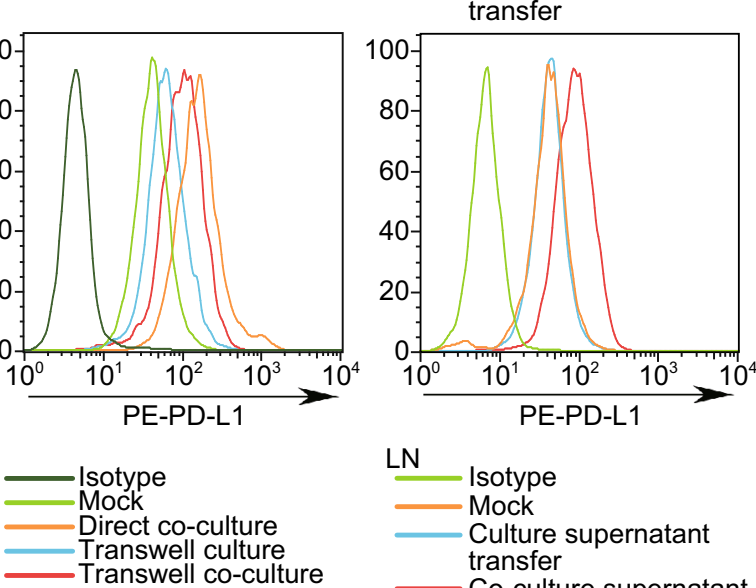

D

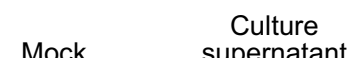

Co-culture supernatant

\begin{tabular}{|l|c|c|c|c|c|c|c|} 
transfer & $\begin{array}{c}\text { transfer } \\
\text { Timmune cells } \\
+ \text { tumor cells }\end{array}$ & $\begin{array}{c}\text { Culture or co-culture } \\
\text { for } 48 \mathrm{~h}\end{array}$ \\
\hline
\end{tabular}

Supernatant

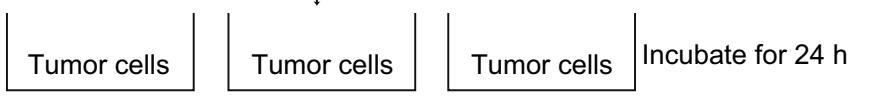

E

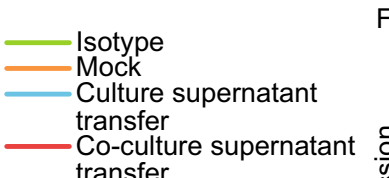

F Mock

- Culture supernatant transfer

Co-culture supernatant transfer

$\frac{\text { 응 }}{\mathrm{N}} 25$
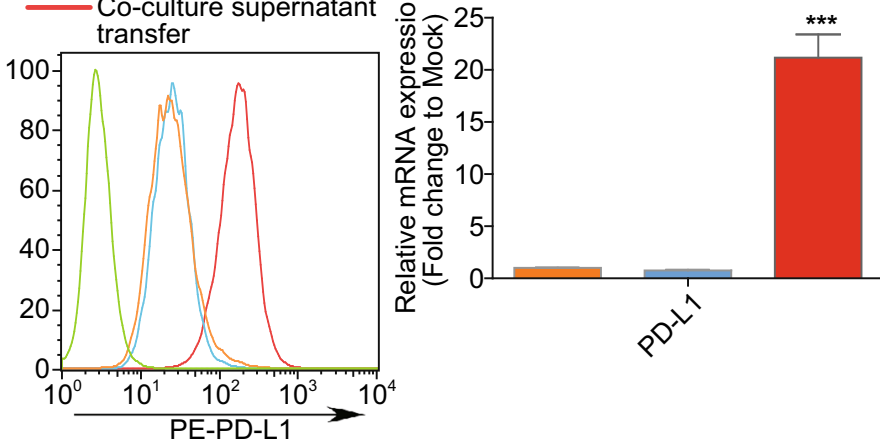

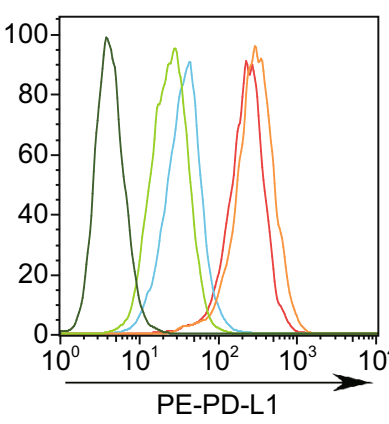

I

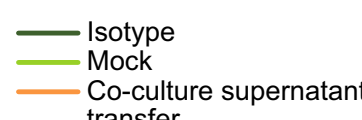
transfer

Supernatant transfer from B16F10 lysate

Supernatant transfer from splenocytes treated B16F10 lysate

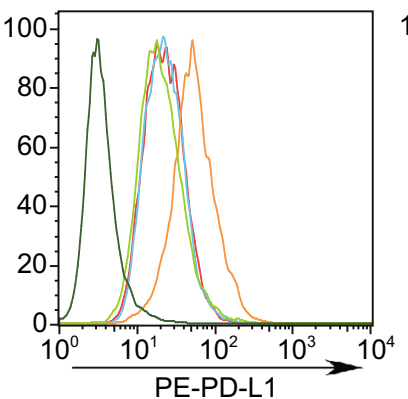

were performed and the same result was obtained (Fig. S1).

Some evidence suggests that cellular components such as tumor cell-derived antigen or other cellular components may also induce PD-L1 expression. To examine these possibilities, we tested whether B16F10 cell-related tumor antigen can stimulate immune cells to secrete type I IFN and whether immune cell-derived components can stimulate tumor cells to upregulate PD-L1. Thus, living immune cells were cultured with B16F10 lysate and live B16F10 tumor cells were cultured with splenocyte lysate. We found that neither lysate can induce PD-L1 expression (Fig. 1I and 1J). These results demonstrated that cell lysate is not sufficient to upregulate PD-L1, suggesting that living cells are required. 
Figure 1. Upregulation of PD-L1 in tumor cells required secreted factors from living cells after direct cell-cell interactions. (A) Schematic diagram of the different co-culture conditions of tumor cells and immune cells (primary splenocytes, bone marrow (BM)-derived cells, or lymph node (LN)derived cells). Tumor cells were directly mixed with immune cells (Direct co-culture) or not (Mock). In the transwell co-culture system, tumor cells were seeded onto the upper insert with the lower compartment containing immune cells (Transwell culture) or a mixture of immune cells and tumor cells (Transwell coculture). (B and C) Expression of PD-L1 in B16F10 cells was determined by flow cytometry (B) and RT-qPCR (C). (D) Schematic diagram for treatment of tumor cells with supernatant from co-cultured tumor cells and splenocytes (Co-culture supernatant transfer), tumor cells alone (Mock) or splenocytes alone (Culture supernatant transfer) as control groups. ( $E$ and F) Expression of PD-L1 was determined by flow cytometry (E) and RT-qPCR (F). (G and H) PD-L1 expression was determined by flow cytometry in B16F10 cells by coculturing with BM $(\mathrm{G})$ or $\mathrm{LN}$ cells $(\mathrm{H})$. (I) B16F10 tumor cells were treated for $24 \mathrm{~h}$ with supernatant from a $48 \mathrm{~h}$ culture of live B16F10 cells (Mock), live splenocytes with B16F10 lysate (supernatant transfer from splenocytes treated B16F10 lysate), live B16F10 cells and live splenocytes (Co-culture supernatant transfer), or B16F10 cell lysate (supernatant transfer from B16F10 lysate). (J) Similarly, B16F10 tumor cells were treated with supernatant from live B16F10 cells with splenocyte lysate (supernatant transfer from B16F10 treated splenocytes lysate), or splenocyte lysate (supernatant transfer from splenocytes lysate). PD-L1 expression was measured by flow cytometry.

It has been reported that PD-L1 expression is induced by IFN signaling. Here we confirmed that the interferon signal was involved. The mRNA expression level of interferon stimulated genes such as IRF7 and ISG15 was significantly upregulated by the supernatant derived from the co-culture of B16F10 cells with bone marrow cells, lymph node cells, or splenocytes (Fig. 2A-C). Moreover, the phosphorylation of STAT1 and STAT3 were increased by supernatant treatment (Fig. 2D). Further, it was observed that co-culture of B16F10 and immune cells contributed to more IFN- $\alpha$ and IFN- $\beta$ release in their supernatant (Fig. S2A and S2B). It is known that interferons (IFN- $\alpha$, IFN- $\beta$, and IFN- $\gamma$ ) induce PD-L1 in tumor cells and the above data suggested that interferon signaling may mediate PD-L1 expression in this system. To determine whether the expression of PD-L1 can be induced by type I IFN in B16F10 cells, we treated B16F10 cells with IFN- $\beta$ and the flow cytometry results showed that IFN- $\beta$ can induce PD-L1 in a concentration-dependent manner (Fig. S3A). Additionally, by using human fibrosarcoma $2 \mathrm{fTGH}$ cells (ifnar2+/+) and the mutant U5A cells (ifnar2-/-) that lack of type I interferon receptor subunit ifnar2, we found that IFN- $\alpha$ induced PD-L1 in $2 \mathrm{fTGH}$ cells, but failed to induce PD-L1 in U5A cells (Fig. S3B). These data suggested that the induction of PD-L1 by co-culture supernatant may be interferon receptor dependent. To test this hypothesis, antiIFNAR1 antibody was added to the co-culture medium. We found that the neutralizing antibody reduced splenocyte-induced PD-L1 expression in B16F10 cells (Fig. 2E). The suppression of PD-L1 expression by anti-IFNAR1 was further confirmed by transwell and supernatant transfer assays (Fig. 2F and 2G). Similarly, these results were observed in $\mathrm{BM}$ and LN cell co-culture experiments (Fig. S4). However, in splenocytes and LN cell co-culture, anti-IFNAR1 antibody only partially blocked PD-L1 expression, suggesting that other secreted factors were involved in stimulating the PD-L1 expression. After testing the different supernatant by cytokines/chemokines microarray, higher IFN-y, IL-6 (well proved for PD-L1 regulation), and G-CSF, Leptin, MIG, MCP5, MIP-1a (firstly observed here may related to PD-L1 regulation) expression were found in the supernatant from coculture of B16F10 and immune cells compared to supernatant from B16F10 (Figs. 2H, S5A, and S5B). Additionally, we found that after adding the anti-IFNAR1 antibody, PD-L1 expression in B16F10 cells were unable to response to type I IFN stimulation (Fig. S3C). However, IFN-y still induced PD$\mathrm{L} 1$ expression in B16F10 cells treated with and without antiIFNAR1 antibody (Fig. S3D). The above findings suggested a working model in which direct contaction between immune cells and tumor cells induces the secretion of factors including type I/II interferons, then these factors promote PD$\mathrm{L} 1$ expression in both contacted and uncontacted tumor cells (Fig. 2l).

PD-L1 is expressed in multiple different tumor cells that suppress anti-tumor immunity in the tumor microenvironment. By binding to its receptor PD-1 on T cells, PD-L1 inhibits the T cell-mediated immune response. Targeting PDL1/PD-1 signaling has been shown to be a highly efficacious cancer immunotherapy (Brahmer et al., 2012; Topalian et al., 2012). Several signaling pathways have been shown to upregulate PD-L1 in tumor cells, including NF-KB, AKT, IFN, and IL-6 (Chen et al., 2012; Jin et al., 2013; Gowrishankar et al., 2015; Lastwika et al., 2016). However, the factors promoting elevated PD-L1 expression in the tumor microenvironment have not been thoroughly elucidated.

In this study, we demonstrated that tumor cell PD-L1 expression is elevated by co-culture with immune cells, and this effect is dependent on the direct interaction between tumor and immune cells. A previous study revealed that CD11 $\mathrm{b}^{+}$myeloid cells adhered to the surface of tumor cells to induce tumor PD-L1 expression through the p38 pathway (Noh et al., 2015). Here, we found that direct cell-cell interactions are required for the production of the secreted factors that induce PD-L1 expression in B16F10 cells. Additionally, the secretome including IFNs induce the PD-L1 expression. However, PD-L1 expression can be partially blocked with anti-IFNAR1 antibody in splenocytes and LN cells co-culture models. These data suggested the possible contribution of other molecules in the regulation of PD-L1 expression by protein microassay. But in our study, which cell population secreted IFNs and which type of immune 

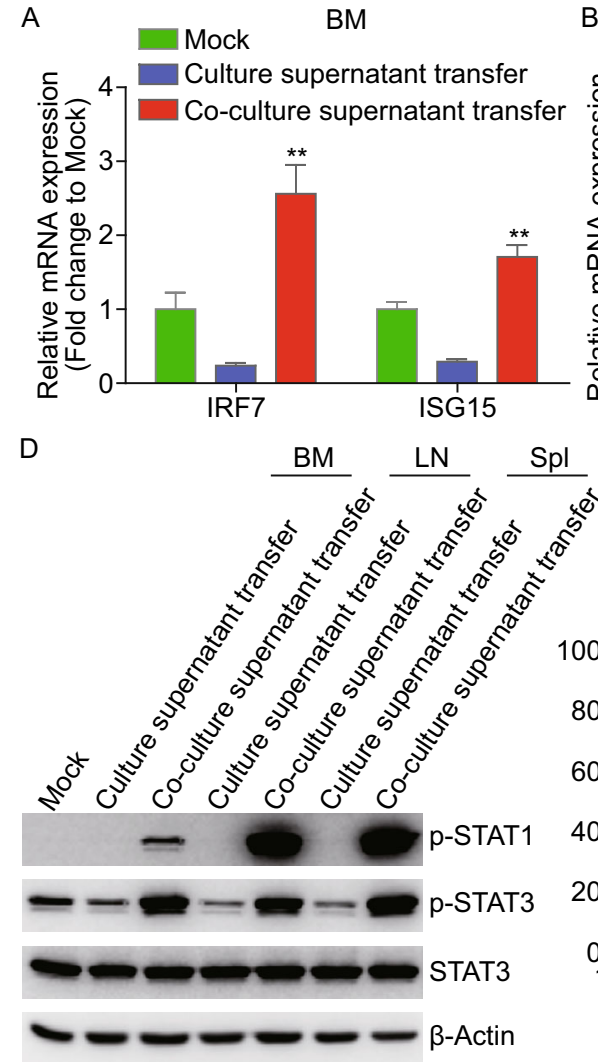

$\mathrm{H}$
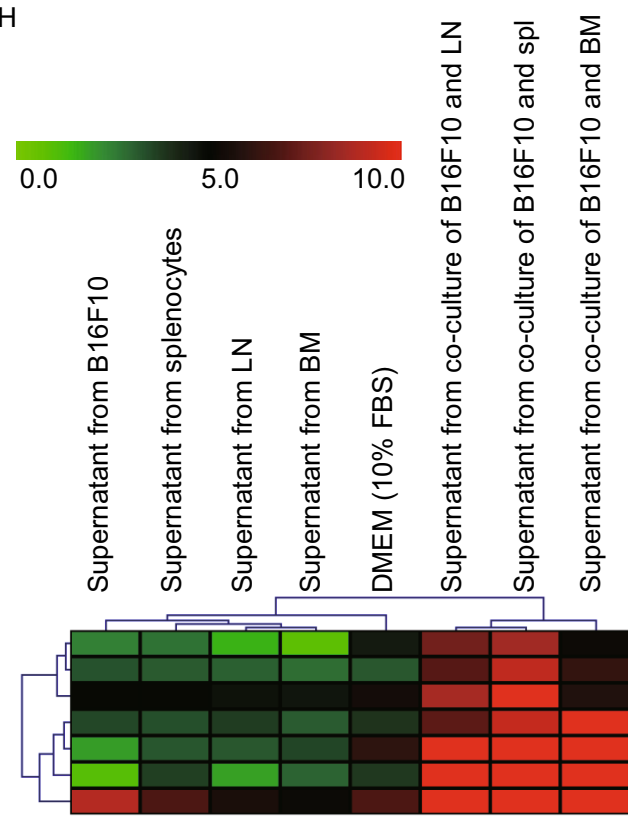
Leptin MIG IFN-Y G-CSF MIP-1a IL-6

cells play a key point for cell-cell contaction still need further study.

On the basis of others' and our studies, we think that $\mathrm{CD}_{11 \mathrm{~b}^{+}}$cells may phagocytose tumor cells to trigger production of type I IFN in STING signaling-dependent manner.

Blockade of PD-1/PD-L1 signaling with monoclonal antibodies has been demonstrated to be a promising new
LN

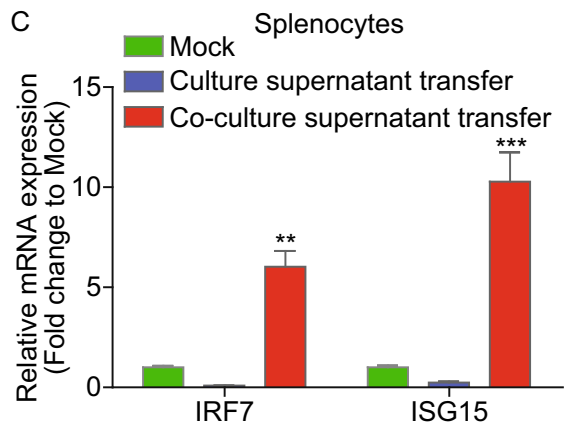

$\mathrm{F}$

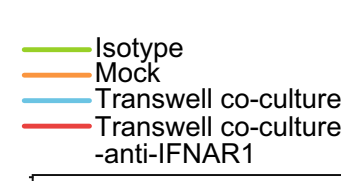

$\mathrm{G}$
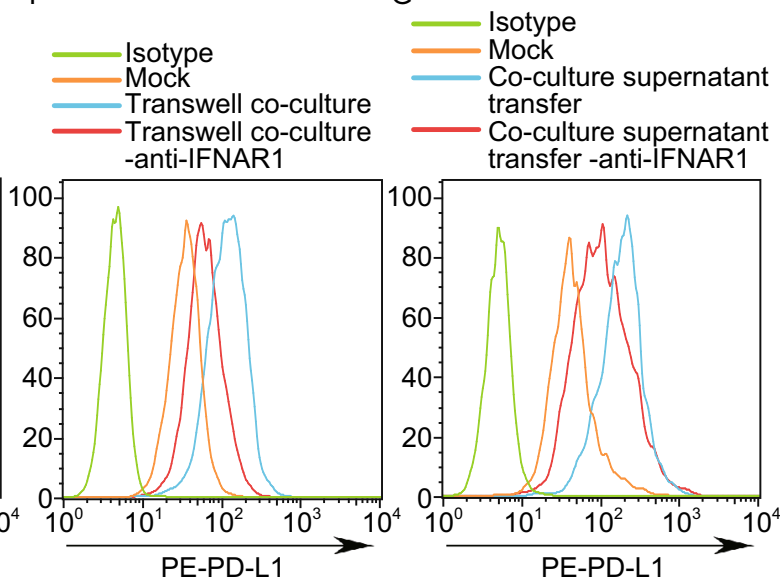

I

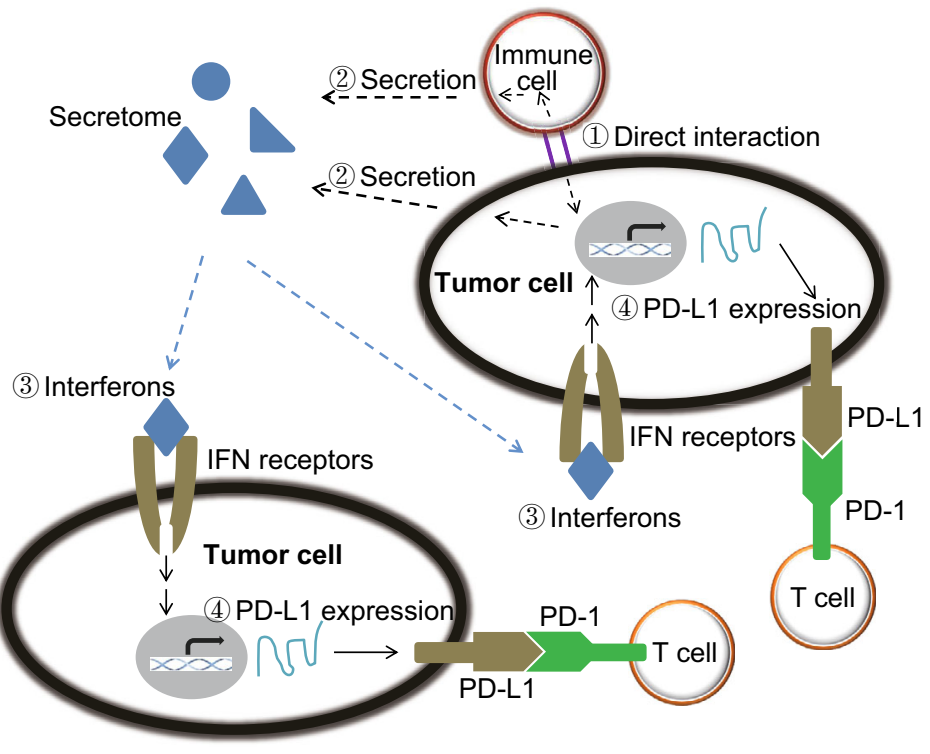

immunotherapy. However, this therapy has a limited role on extending survival time and can lead to inflammatory side effects called immune-related adverse events (irAEs) in other normal tissues (Brahmer et al., 2012). Our findings suggest an alternative way to find specific inhibitors to limit PD-L1 expression in tumor cells by blocking its up-stream induced signaling. Such screened inhibitors could be used in 
Figure 2. Upregulation of PD-L1 in tumor cells was dependent on IFNAR1 signaling. (A-C) IRF7 and ISG15 mRNA level in B16F10 cells were determined, which were treated for $24 \mathrm{~h}$ with co-culture supernatant from B16F10 cells and bone marrow (A), or lymph nodes derived cells $(B)$ or splenocytes (C), respectively. (D) After treated with the similar above supernatant in $(A-C)$ for $15 \mathrm{~min}$, the phosphorylation of STAT1 and STAT3 were determined. (E-G) B16F10 cells were cocultured with splenocytes in three different co-culture systems with or without the $20 \mu \mathrm{g} / \mathrm{mL}$ anti-IFNAR1 antibody. PD-L1 expression was measured by flow cytometry in direct co-culture system (E), transwell co-culture system $(F)$, and supernatant transfer system (G). (H) Heat map demonstrated relative abundance of cytokines in different cell co-culture supernatant. (I) Schematic model of PD-L1 induction in tumor cells by the interaction between immune and tumor cells. Immune cells directly contact tumor cells (1) Direct interaction). Then the secretome is actively secreted into the supernatant (2) Secretion). Interferons, one type of factors in the secretome, bind to IFN receptors (3) Interferons) and further upregulate endogenous PD-L1 expression in both contacted tumor cells and uncontacted tumor cells (4) PD-L1 expression).

combination with PD-L1 antibodies to achieve better effect for cancer therapy.

\section{FOOTNOTES}

The mouse melanoma B16F10 cells were a gift from Prof. Hongyan Wang. The mouse colorectal cancer MC38 cells were kindly provided by Prof. Serge Fuchs. The anti-IFNAR1 antibody was a gift from Prof. Jianghuai Liu. We are very grateful that U5A, $2 \mathrm{fTGH}$ cell lines were supplied by Professor Zhengfan Jiang and Professor George R. Stark.

This work was supported by Sichuan Science and Technology Project (2013ZZ0004), Shanghai Institutes for Biological Science, Chinese Academy of Sciences \& Sichuan Huiyang Life Science and Technology Corp. Research Program (Y363S21763), the National Basic Research Program (973 Program) (Nos. 2011CB510104 and 2014CB964704), Zhejiang Sci-Tech University Grant (1204807-Y), and grant from the Sino-American Joint Laboratory between Conba Group and Zhejiang Sci-Tech University.

Yuan-Qin Yang, Wen-Jie Dong, Xiao-Fei Yin, Yan-Ni Xu, Yu Yang, Jiao-Jiao Wang, Su-Jing Yuan, Jing Xiao, Jonathan Howard DeLong, Liang Chu, Hai-Neng Xu, Xiu-Mei Zhou, Ru-Wei Wang, Ling Fang, Xin-Yuan Liu, and Kang-Jian Zhang declare that they have no conflict of interest. All institutional and national guidelines for the care and use of laboratory animals were followed.

Yuan-Qin Yang and Wen-Jie Dong contributed equally to this work.

Electronic supplementary material The online version of this article (doi:10.1007/s13238-016-0281-6) contains supplementary material, which is available to authorized users.
Yuan-Qin Yang ${ }^{1}$, Wen-Jie Dong ${ }^{1}$, Xiao-Fei Yin ${ }^{2}$, Yan-Ni Xu ${ }^{4}$, Yu Yang ${ }^{5}$, Jiao-Jiao Wang ${ }^{1}$, Su-Jing Yuan ${ }^{2}$, Jing Xiao ${ }^{2}$, Jonathan Howard DeLong ${ }^{6}$, Liang $\mathrm{Chu}^{2}$, Hai-Neng $\mathrm{Xu}^{7}$, XiuMei Zhou ${ }^{1}$, Ru-Wei Wang ${ }^{8}$, Ling Fang ${ }^{8}$, Xin-Yuan Liu ${ }^{1,2}{ }^{(D)}$, Kang-Jian Zhang ${ }^{2,3 凶}$ (D)

${ }^{1}$ Xinyuan Institute of Medicine and Biotechnology, Zhejiang SciTech University, Hangzhou 310018, China

2 State Key Laboratory of Cell Biology, Shanghai Institute of Biochemistry and Cell Biology, Shanghai Institutes for Biological Sciences, Chinese Academy of Sciences, Shanghai 200031, China

${ }^{3}$ Sichuan Huiyang Life Science and Technology Corp., Chengdu 610021, China

${ }^{4}$ College of Life Sciences, Northwest Agriculture and Forestry University, Yangling 712100, China

${ }^{5}$ Central China Normal University, Wuhan 430079, China

${ }^{6}$ Department of Pathobiology, School of Veterinary Medicine, University of Pennsylvania, Philadelphia, PA 19104, USA

7 Department of Radiation Oncology, University of Pennsylvania Perelman School of Medicine, 3400 Civic Center Blvd., Philadelphia, PA 19104, USA

${ }^{8}$ Zhejiang Conba Pharmaceutical Co., Ltd, Hangzhou 310018, China

$\triangle$ Correspondence: xyliu@sibcb.ac.cn (X.-Y. Liu), zhangkangjian@sibcb.ac.cn (K.-J. Zhang)

\section{OPEN ACCESS}

This article is distributed under the terms of the Creative Commons Attribution 4.0 International License (http://creativecommons.org/ licenses/by/4.0/), which permits unrestricted use, distribution, and reproduction in any medium, provided you give appropriate credit to the original author(s) and the source, provide a link to the Creative Commons license, and indicate if changes were made.

\section{REFERENCES}

Brahmer JR, Tykodi SS, Chow LQ, Hwu WJ, Topalian SL, Hwu P, Drake CG, Camacho LH, Kauh J, Odunsi K et al (2012) Safety and activity of anti-PD-L1 antibody in patients with advanced cancer. N Engl J Med 366:2455-2465

Chen J, Feng Y, Lu L, Wang H, Dai L, Li Y, Zhang P (2012) Interferon-gamma induced PD-L1 surface expression on human oral squamous carcinoma via PKD2 signal pathway. Immunobiology 217:385-393

Cho YA, Yoon HJ, Lee JI, Hong SP, Hong SD (2011) Relationship between the expressions of PD-L1 and tumor-infiltrating lymphocytes in oral squamous cell carcinoma. Oral Oncol 47:11481153

Ejlerskov P, Hultberg JG, Wang J, Carlsson R, Ambjorn M, Kuss M, Liu Y, Porcu G, Kolkova K, Friis Rundsten C et al (2015) Lack of neuronal IFN- $\beta$-IFNAR causes lewy body-and parkinson's disease-like dementia. Cell 163:324-339

Furuta J, Inozume T, Harada K, Shimada S (2014) CD271 on melanoma cell is an IFN- $\gamma$-inducible immunosuppressive factor that mediates downregulation of melanoma antigens. J Investig Dermatol 134:1369-1377 
Gowrishankar K, Gunatilake D, Gallagher SJ, Tiffen J, Rizos H, Hersey $P$ (2015) Inducible but not constitutive expression of PDL1 in human melanoma cells is dependent on activation of NFKB. PLoS One 10:e0123410

Hou J, Yu Z, Xiang R, Li C, Wang L, Chen S, Li Q, Chen M, Wang L (2014) Correlation between infiltration of FOXP3+ regulatory $T$ cells and expression of $\mathrm{B} 7-\mathrm{H} 1$ in the tumor tissues of gastric cancer. Exp Mol Pathol 96:284-291

Jin YH, Hou W, Kang HS, Koh CS, Kim BS (2013) The role of interleukin- 6 in the expression of PD-1 and PDL-1 on central nervous system cells following infection with Theiler's murine encephalomyelitis virus. J Virol 87:11538-11551

Joyce JA, Fearon DT (2015) T cell exclusion, immune privilege, and the tumor microenvironment. Science 348:74-80

Kronig H, Kremmler L, Haller B, Englert C, Peschel C, Andreesen R, Blank CU (2014) Interferon-induced programmed death-ligand 1 (PD-L1/B7-H1) expression increases on human acute myeloid leukemia blast cells during treatment. Eur J Haematol 92:195203

Lastwika KJ, Wilson W 3rd, Li QK, Norris J, Xu H, Ghazarian SR, Kitagawa H, Kawabata S, Taube JM, Yao S et al (2016) Control of
PD-L1 expression by oncogenic activation of the AKT-mTOR pathway in non-small cell lung Cancer. Cancer Res 76:227-238 McClanahan F, Riches JC, Miller S, Day WP, Kotsiou E, Neuberg D, Croce CM, Capasso M, Gribben JG (2015) Mechanisms of PDL1/PD-1-mediated CD8 T-cell dysfunction in the context of agingrelated immune defects in the $\mathrm{E} \mu-\mathrm{TCL} 1 \mathrm{CLL}$ mouse model. Blood 126:212-221

Noh H, Hu J, Wang X, Xia X, Satelli A, Li S (2015) Immune checkpoint regulator PD-L1 expression on tumor cells by contacting CD11b positive bone marrow derived stromal cells. Cell Commun Signal 13:14

Topalian SL, Hodi FS, Brahmer JR, Gettinger SN, Smith DC, McDermott DF, Powderly JD, Carvajal RD, Sosman JA, Atkins MB et al (2012) Safety, activity, and immune correlates of antiPD-1 antibody in cancer. N Engl J Med 366:2443-2454

York AG, Williams KJ, Argus JP, Zhou QD, Brar G, Vergnes L, Gray EE, Zhen A, Wu NC, Yamada DH et al (2015) Limiting cholesterol biosynthetic flux spontaneously engages type I IFN signaling. Cell 163:1716-1729 